\title{
Aprovechamiento de la madera de encino en México
}

\author{
Carmen de la Paz Pérez Olvera ${ }^{1}$ \\ Raymundo Dávalos Sotelo ${ }^{2}$ \\ Estela Guerrero Cuacuil ${ }^{1}$
}

\begin{abstract}
RESUMEN
Se hace una descripción de la situación del uso de la madera de encino en México. Se incluyen las estadísticas de existencias maderables y de producción más recientes. Se hace referencia a la distribución de este importante recurso en el país. Los temas tratados son: utilización de la madera de encino (incluyendo celulosa, escuadría, combustible, durmientes, postes, pilotes, morillos, chapa y madera contrachapada) y la influencia de las características tecnológicas en su uso. Se discute el aprovechamiento de la madera de encino en México en el contexto del panorama internacional. Se concluye que es una tarea urgente buscar la conservación de los encinares para preservar el equilibrio ecológico de los sitios donde crecen.
\end{abstract}

PALABRAS CLAVE:

Aprovechamiento, madera, encino, México

\section{INTRODUCCIÓN}

El aprovechamiento adecuado de cualquier recurso natural se traduce en una fuente de riqueza y mejoramiento económico para el país que sepa utilizarlo. En el caso del recurso forestal maderable, por ser renovable, es inagotable, siempre y

\begin{abstract}
A description is made of the situation of oak wood use in México. The most recent statistics of standing forest volume and wood production are included. Reference is made of the distribution of this resource in the country. The subjects treated in this work are: utilization of oak wood (including cellulose, sawn wood, fuel, crossties, poles, piles, round wood, veneer and plywood), and the influence of the technological characteristics on its use. The exploitation of oakwood is discussed in the context of the international market.It is concluded that the conservation of oak forests is an urgent task to preserve the ecological equilibrium of its growing areas.
\end{abstract}

KEY WORDS:

Exploitation, wood, oak, México.

cuando su manejo sea racional. El territorio mexicano tiene una superficie continental de 195 millones de ha, de las cuales 30.4 corresponden a bosques y 26.4 a selvas (SEMARNAP, 1998). Dentro de esta área forestal, los encinos, como comúnmente se conoce en México al conjunto de especies del género Quercus, constituyen el recurso

1 Universidad Autónoma Metropolitana Iztapalapa. División de Ciencias Biológicas y de la Salud. Departamento de Biología. Av. Michoacán y La Purísima s/n. 09340. México, D. F.

2 Instituto de Ecología, A. C. Departamento de Productos Forestales y Conservación de Bosques. Km 2.5 antigua carretera a Coatepec. Apdo Postal 63. 91000 Xalapa, Ver. c.e.davalos@ecologia.edu.mx Manuscrito recibido el 15 de octubre de 1999. Aceptado el 23 de febrero de 2000. 
maderable más abundante, después de los pinos, básicamente en los bosques de clima templado del país, aunque también se encuentran representantes tropicales como $Q$. oleoides y $Q$. sapoteifolia que crecen incluso al nivel del mar (Zavala, 1995; Pennington y Sarukhán, 1998; Aguilar et al., 1999) y en las regiones semiáridas (Rzedowski, 1978).

McVaugh (1974) menciona que ningún otro género, con excepción de Pinus, es tan abundante en la vegetación forestal maderable de los sitios montañosos y escarpados de clima templado donde alcanza su máximo desarrollo. Rzedowski (1978) indica que constituye el elemento dominante de la vegetación de la mayoría de las sierras que forman el territorio mexicano y que es en el Eje Neovolcánico en donde se presenta el mayor número de taxa. Las especies de Quercus se encuentran en todos los estados de la República Mexicana, excepto en Yucatán (Zavala, 1990) (Fig.1).

El género Quercus pertenece a la familia Fagaceae y de acuerdo con sus características morfológicas, Trelease (1924) lo divide en seis subgéneros, tres de los cuales vegetan en México: Leucobalanus o encinos blancos, Erythrobalanus o encinos rojos y Protobalanus o encinos negros. Tanto las especies de los encinos blancos como las de los encinos rojos, se encuentran ampliamente distribuidas en México, con alrededor de 57 para los blancos y 54 para los rojos. Los encinos negros, están restringidos al Norte de Baja California y Norte de Sonora, con cuatro especies, una de ellas arbórea (Aguilar et al., 1999).

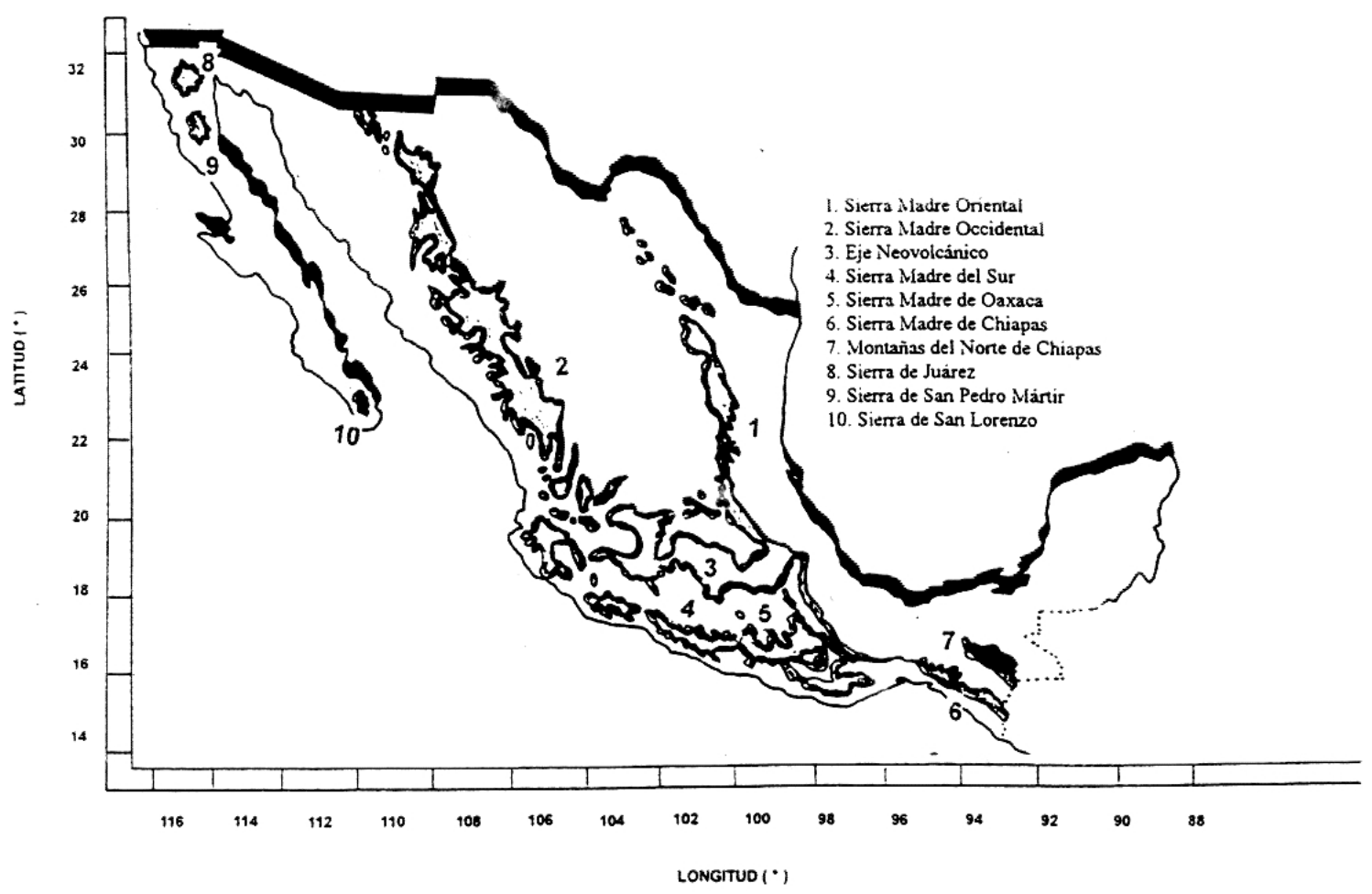

Figura 1. Distribución de los encinos en México. 
Fisonómica y estructuralmente, los encinos presentan dos formas de vida: arbustos y árboles. Estos últimos constituidos por especies cuyas dimensiones varían entre 15 y 30 y hasta 40 $\mathrm{m}$ de altura, con diámetros de $30 \mathrm{a} 60 \mathrm{~cm}$ y en ocasiones hasta de un metro. Algunas de ellas son las que se emplean en el aprovechamiento de la madera de encino en México.

\section{DATOS RECABADOS}

Con base en la bibliografía consultada (SEMARNAP, 1998), en visitas a fábricas y madererías y pláticas con carpinteros, artesanos, campesinos y funcionarios de la SEMARNAP, se obtuvieron los datos que se presentan del aprovechamiento de la madera de encino y de la problemática involucrada en el mismo.

Los conceptos de la influencia de algunas propiedades tecnológicas en su uso, están basados en el trabajo experimental que han realizado los dos primeros autores en especies de encinos mexicanos. Dentro de los productos maderables se incluye a la celulosa, ya que a nivel industrial y comercial y en los anuarios estadísticos está considerada como tal (SEMARNAP, 1998, 1999). Los resultados se presentan en cuadros, gráficas y fotografías.

\section{RESULTADOS}

Productos elaborados. La madera de encino ocupa el segundo lugar de aprovechamiento a nivel nacional $(578,687$ $\mathrm{m}^{3}$ por año), después del pino $(5,783,299$ $\mathrm{m}^{3}$ por año) y antes de las maderas tropicales $\left(169,230 \mathrm{~m}^{3}\right.$ por año) (SEMARNAP 1998), representando el 9\% del total explotado (Fig. 2).

Los estados con mayor aprovechamiento son: Michoacán, Durango,

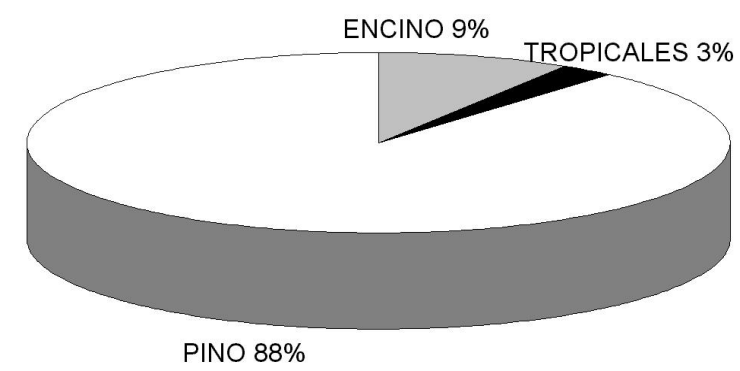

Figura 2. Porcentaje de la producción maderable para encino, pino y tropicales.

Chihuahua, Jalisco, Guanajuato, Hidalgo, Puebla, Sonora, Veracruz y Oaxaca (SEMARNAP, 1998), coincidiendo con Zavala (1995) en cuanto a que son los estados que presentan mayor número de especies, faltando únicamente Guerrero (Fig. 3, Tabla 1).

Los estados que no tienen datos de producción maderable de encino, a pesar de tener representantes de ellos en su territorio son: Baja California Sur, Campeche, Coahuila, Morelos, Quintana Roo, Sinaloa, Tabasco y el Distrito Federal.

Los usos a los que se destina principalmente la madera son: celulosa, escuadría, combustible (leña y carbón), postes, pilotes y morillos, durmientes, chapa y madera contrachapada (triplay) (SEMARNAP, 1998) (Fig. 4, Tabla 2).

\section{Destino de los productos.}

Celulosa. La mayoría de la celulosa se destina a la elaboración de papel kraft o de empaque para trabajo pesado como sacos para el transporte de materiales para construcción (cal, cemento y yeso), para alimento para mascotas y alimentación 
humana, como harina y para la venta de carbón. Otro producto es el cartón que es vendido en láminas o transformado en cajas de diversas dimensiones. Un destino adicional de la celulosa, para envolturas de tipo ligero como bolsas o papel de estraza, empleado además en la manufactura de toallas para las manos y papel revolución y papel periódico.

La celulosa de encino siempre se usa en mezclas con otras especies, principalmente de coníferas; dependiendo del uso es el porcentaje de las mezclas. La mayoría no es sometida a blanqueo.

Escuadría. El producto primario de la escuadría o madera aserrada, se refiere a la obtención de tablas y tablones de diferentes dimensiones, vigas, polines, durmientes y gualdras (Fig. 5), que pueden ser usados como tales en varios usos principalmente en la construcción o ser transformados en variados objetos como: piezas talladas, molduras, cabos y

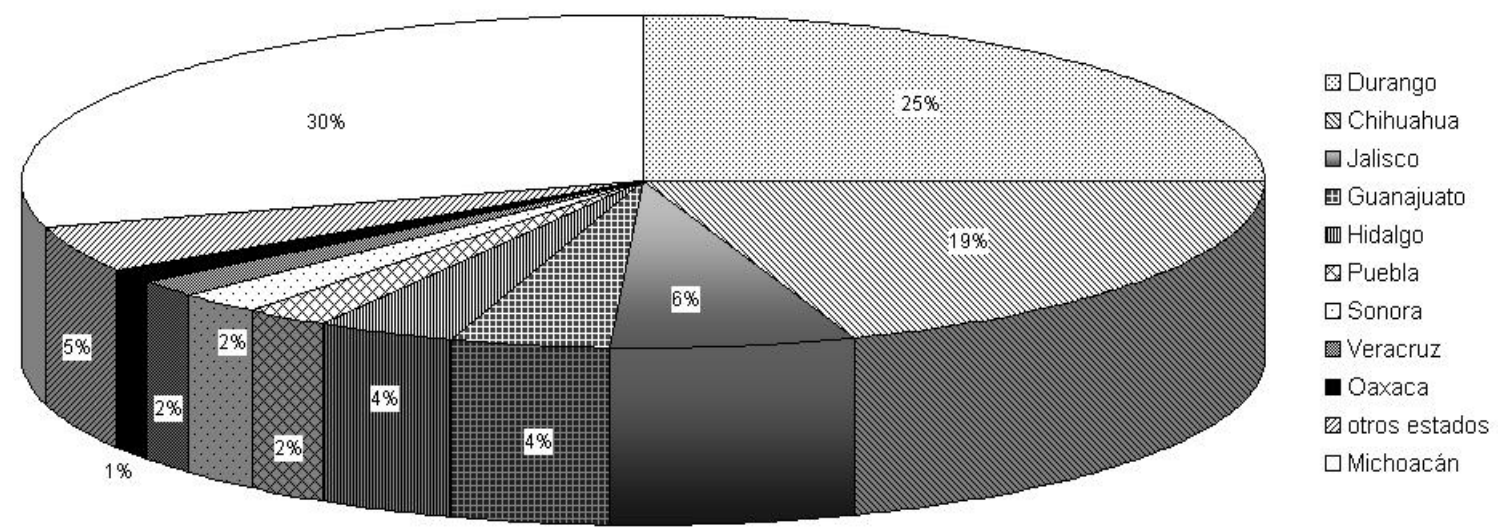

Figura 3. Porcentaje de la producción maderable de encino por estado.

Tabla 1. Producción maderable de encino por estado $\left(\mathrm{m}^{3}\right)$

\begin{tabular}{||l|r|l|r|l|r||}
\hline \multicolumn{1}{|c|}{ ESTADOS } & \multicolumn{1}{c|}{$\mathrm{m}^{3}$} & ESTADOS & $\mathrm{m}^{3}$ & ESTADOS & $\mathrm{m}^{3}$ \\
\hline Aguascalientes & 3,817 & Hidalgo & 20,538 & Querétaro & 215 \\
\hline Baja California N. & 455 & Jalisco & 36,482 & San Luis Potosí & 6,370 \\
\hline Colima & 213 & México & 946 & Sinaloa & 271 \\
\hline Chiapas & 194 & Michoacán & 169,908 & Sonora & 12,757 \\
\hline Chihuahua & 112,743 & Nayarit & 31 & Tamaulipas & 933 \\
\hline Durango & 145,224 & Nuevo León & 1,847 & Tlaxcala & 1,205 \\
\hline Guanajuato & 24,070 & Oaxaca & 7,210 & Veracruz & 9,402 \\
\hline Guerrero & 4,998 & Puebla & 13,461 & Zacatecas & 5,397 \\
\hline \multicolumn{7}{|r}{} \\
\hline
\end{tabular}


Tabla 2. Destino de los productos maderables de encino por estado $\left(\mathrm{m}^{3}\right)$

\begin{tabular}{|c|c|c|c|c|c|c|c|c|}
\hline \multirow{2}{*}{ ESTADOS } & \multirow{2}{*}{$\begin{array}{l}\text { CELU } \\
\text { LOSA }\end{array}$} & \multirow{2}{*}{$\begin{array}{c}\text { ESCUA } \\
\text { DRIA }\end{array}$} & \multicolumn{2}{|c|}{ 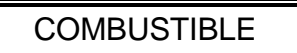 } & \multirow{2}{*}{$\begin{array}{l}\text { MORILLOS, } \\
\text { POSTES Y } \\
\text { PILOTES }\end{array}$} & \multirow{2}{*}{$\begin{array}{c}\text { DURMIEN } \\
\text { TES }\end{array}$} & \multirow{2}{*}{$\begin{array}{l}\text { CHAPA Y } \\
\text { TRIPLAY }\end{array}$} & \multirow{2}{*}{ TOTAL } \\
\hline & & & LEÑA & CARBON & & & & \\
\hline Aguascalientes & & & 3,786 & & 31 & & & 3,817 \\
\hline Baja California N. & & & 455 & & & & & 455 \\
\hline Colima & & & 7 & 142 & 3 & & & 213 \\
\hline Chiapas & & 194 & & & & & & 194 \\
\hline Chihuahua & 109,184 & 2,619 & 491 & 449 & & & & 112,743 \\
\hline Durango & 33,567 & 99,193 & 6,429 & 595 & 6,429 & 65 & & 145,224 \\
\hline Guanajuato & 254 & 6 & 3,651 & 18,885 & 1,274 & & & 24,070 \\
\hline Guerrero & & 4,856 & 30 & 112 & & & & 4,998 \\
\hline Hidalgo & 802 & 15,562 & 2,684 & 1,323 & 155 & 12 & & 20,538 \\
\hline Jalisco & 13,748 & 2,835 & 10,158 & 9,262 & 283 & & 196 & 36,482 \\
\hline México & 83 & 759 & 93 & & 11 & & & 946 \\
\hline Michoacán & 138,328 & 30,582 & & 998 & & & & 169,908 \\
\hline Nayarit & & & & 31 & & & & 31 \\
\hline Nuevo León & & 1,473 & 360 & & & 14 & & 1,847 \\
\hline Oaxaca & & 632 & 5,671 & 851 & 56 & & & 7,210 \\
\hline Puebla & 889 & 8,274 & 4,207 & 20 & 71 & & & 13,461 \\
\hline Querétaro & & & 215 & & & & & 215 \\
\hline San Luis Potosí & & 54 & & 20 & & 6296 & & 6,370 \\
\hline Sinaloa & & 271 & & & & & & 271 \\
\hline Sonora & 7,973 & 80 & 4,704 & & & & & 12,757 \\
\hline Tamaulipas & & 923 & & 10 & & & & 933 \\
\hline Tlaxcala & 55 & 789 & 330 & 29 & 2 & & & 1,205 \\
\hline Veracruz & 211 & 6,441 & 36 & 816 & & & & 9,402 \\
\hline Zacatecas & & & 728 & 405 & 4264 & & & 5397 \\
\hline TOTAL & 306,894 & 175,604 & 42,981 & 33,948 & 12,677 & 6,387 & 196 & 578,687 \\
\hline
\end{tabular}

Fuente: SEMARNAP, 1998 


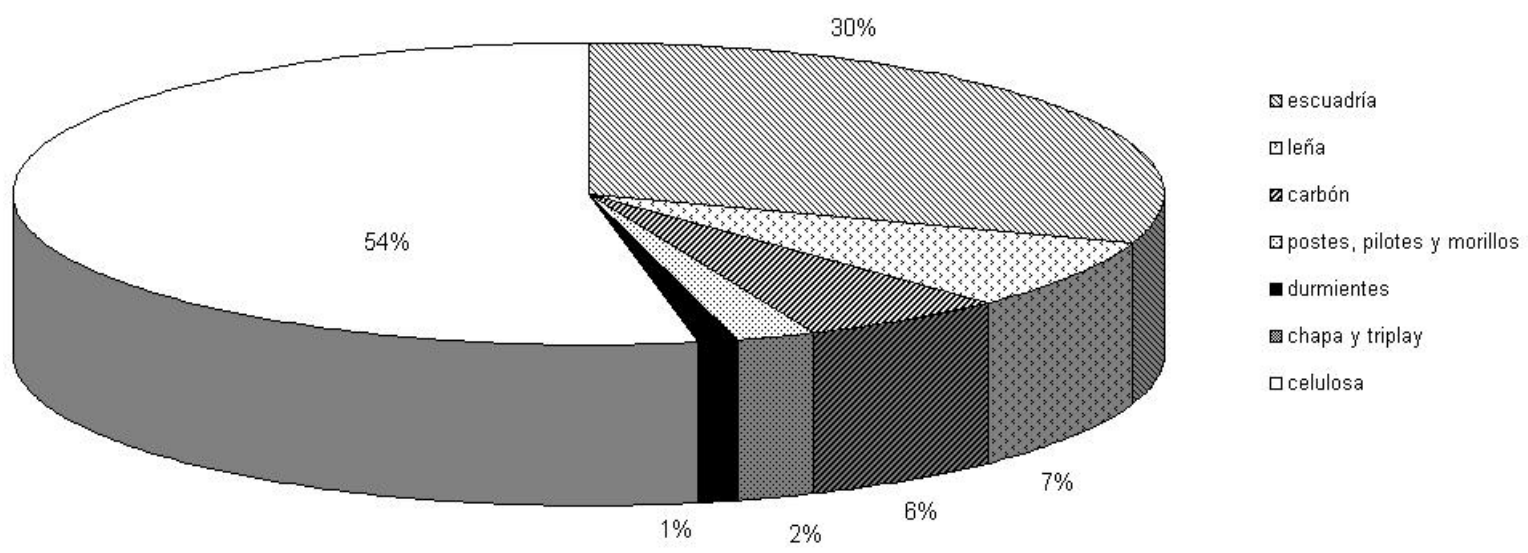

Figura 4. Porcentaje de los productos maderables de encino a nivel nacional.

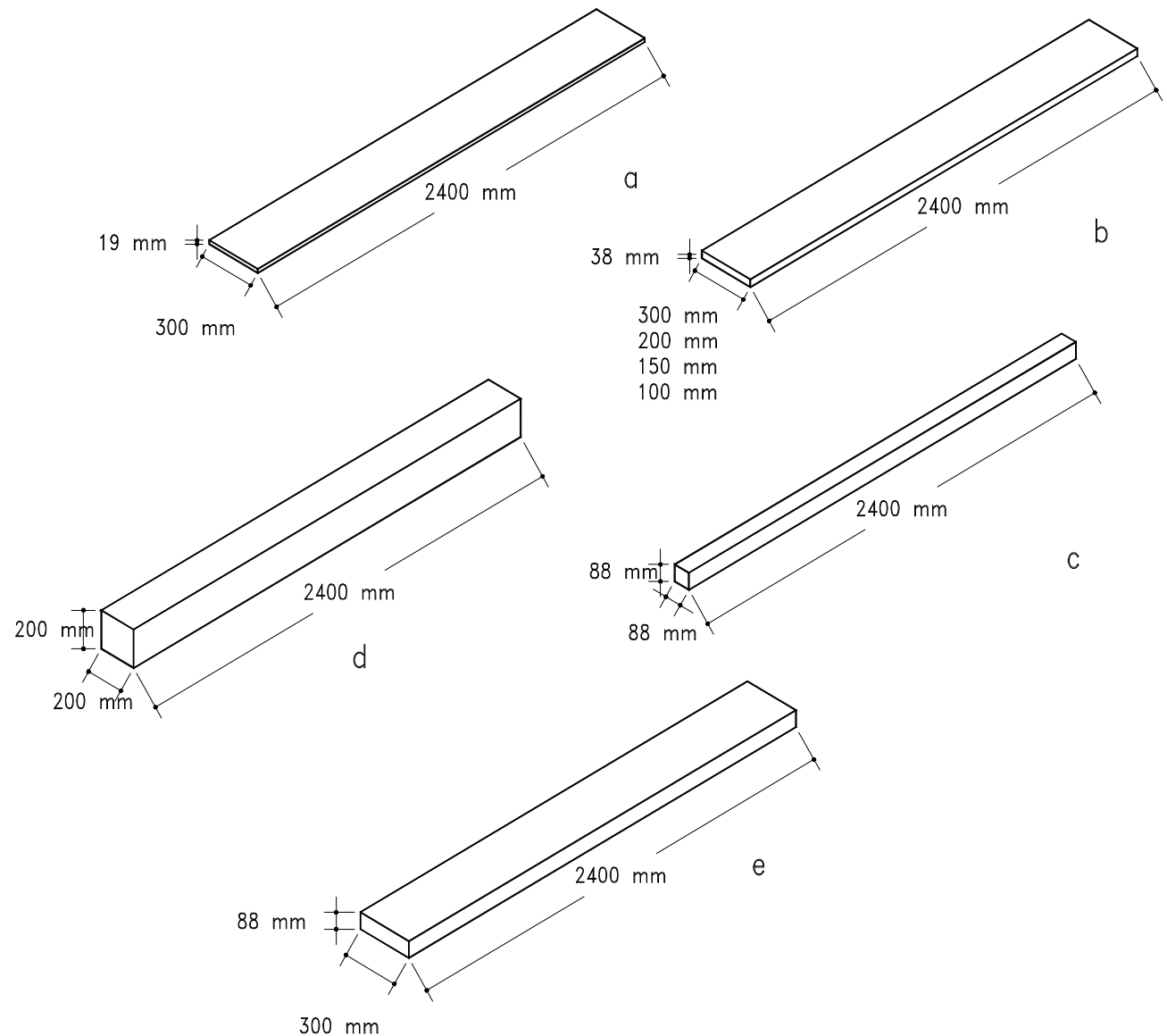

Figura 5. Productos primarios de la escuadría a. tabla. b. tablón. c. polín. d. durmiente. e. viga. 
mangos para herramienta, artículos decorativos en general, marcos de puertas y ventanas, muebles, pisos en forma de duela, parquet y adoquín, techos, lambrines, zoclos, artesanías, en donde Michoacán tiene una producción muy importante (De la Paz Pérez y Quintanar, 1998), utensilios de cocina, juguetes, partes de instrumentos musicales como pianos y guitarras, etc. (Fig. 6).

La escuadría es el uso donde se ponen de manifiesto al máximo las características tecnológicas y anatómicas de esta madera, lo que da la posibilidad de crear objetos de gran calidad y valor estético y comercial, por lo que debería destinarse un mayor porcentaje a este uso, justificando así la transformación de árboles de grandes tallas en estos productos.

Combustible. La madera de encino se usa como combustible de dos formas: directamente como leña o transformándola en carbón, siendo mayor el aprovechamiento en leña (Bravo, 1995). Rzedowski (1978), menciona que grandes extensiones de encinares se han extinguido debido a la explotación desmedida para carbón vegetal.

A pesar de que, tanto la leña como el carbón de encino, son de los mejores combustibles vegetales, se desaprovechan en gran medida tanto las propiedades anatómicas como las tecnológicas que presentan la mayoría de las especies. Los productos de celulosa y carbón, aunque útiles, no justifican la transformación de valiosos árboles de grandes tallas en ellos, por ser de bajo valor agregado, particularmente el carbón.

Durmientes. Son también considerados dentro de la escuadría, la cual actualmente está tomando auge, debido a que los requerimientos van en aumento para dar mantenimiento a las líneas férreas nacionales sobre todo en el norte del país (Becerra, 1999).
Postes, pilotes y morillos. Estos productos se obtienen, aprovechando el fuste comercial de árboles delgados y largos, descortezados o con corteza. Los morillos son utilizados en la construcción rural, como vigas en techos o como refuerzos en las paredes. Los postes se utilizan para sostener las líneas de comunicación de energía eléctrica, así como para cubrir las necesidades de la industria minera.

Chapa y madera contrachapada. En el aprovechamiento para chapa y contrachapados (triplay), se ponen de manifiesto las cualidades estéticas de la madera, producto que únicamente se procesa en el estado de Jalisco. La chapa se obtiene de madera desenrollada, de estructura homogénea que es utilizada para dar acabados a muebles, sobre todo para cubrir los extremos y bordes, dando un terminado fino. El triplay se obtiene de la misma forma que la chapa, pero en este caso las láminas se acomodan en capas en números impares, que generalmente tienen, dimensiones que varían de 3 a $19 \mathrm{~mm} x$ $1.24 \mathrm{~m} \times 2.44 \mathrm{~m}$. Dependiendo del grosor es el uso que se le da, aunque se destina principalmente para la fabricación de muebles.

Todos los productos elaborados se obtienen del fuste de los árboles. En países europeos y de América del Norte, los árboles usados para celulosa son árboles débiles, que son talados para que los árboles vigorosos alcancen su plena madurez para ser utilizados como madera aserrada. También las costeras son utilizadas para pulpa. En Estados Unidos de Norteamérica y en Canadá, la madera es el material de construcción más barato y más versátil, aunque el encino se destina principalmente a otros usos. Sin embargo, el uso de estas especies en la construcción se ha incrementado en años recientes (Green et al., 1994). 


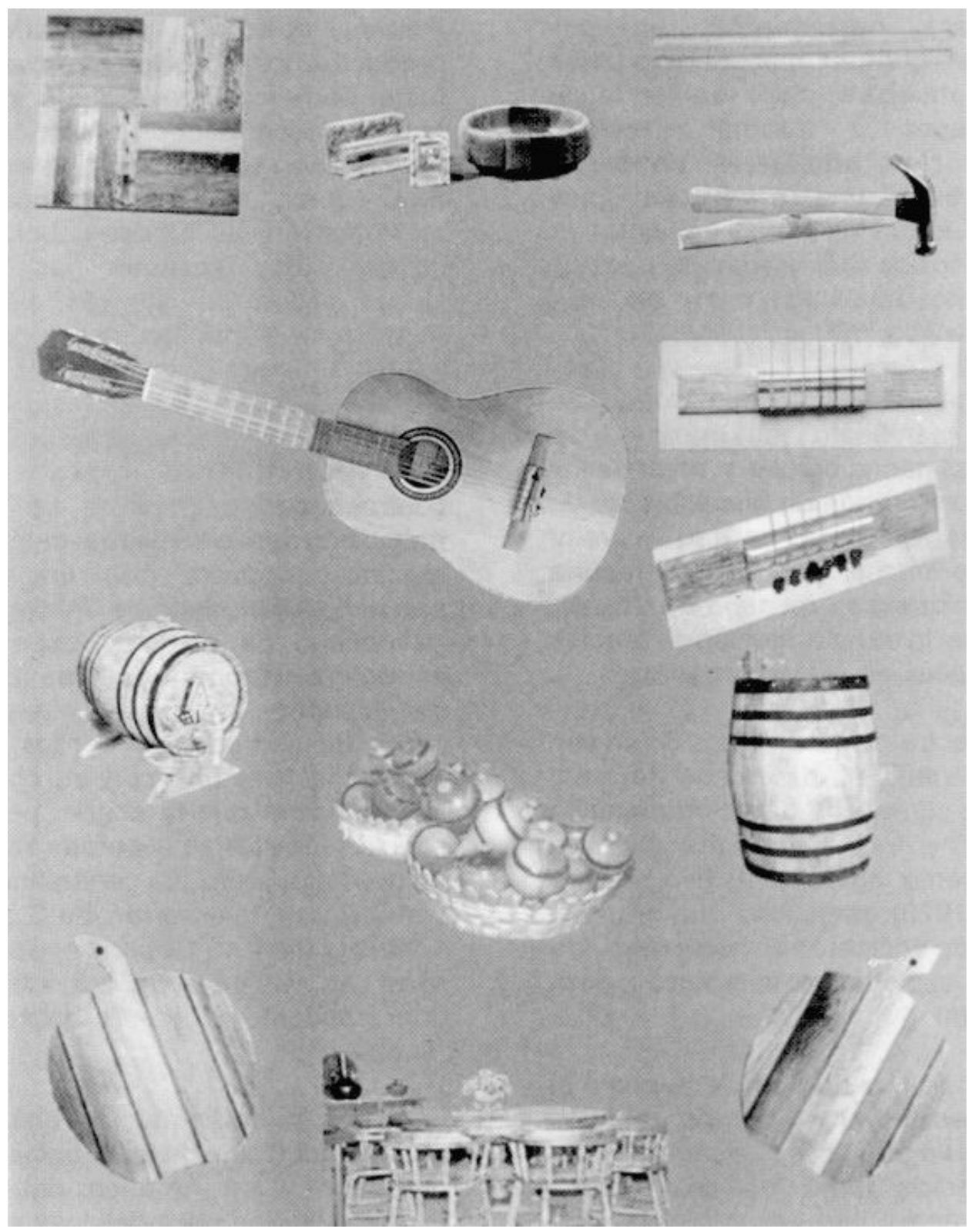

Figura 6. Algunos productos derivados de la escuadría.

\section{INFLUENCIA DE LAS CARACTERÍSTICAS TECNOLÓGICAS EN SU USO}

En el aprovechamiento industrial de la madera de encino en México, la del grupo de los rojos se utiliza más que la del grupo de los blancos. Esto, tal vez se deba a que los encinos rojos son mas abundantes y con mayor distribución que los blancos (Aguilar et al., 1999). Además de que los encinos blancos tienen mayor densidad, mayor dureza, mayor cantidad de cristales y tílides en sus lúmenes, sus rayos multiseriados son más anchos y más altos que los de los encinos rojos, por lo que son más susceptibles a los cambios dimensionales (contracciones e hinchamientos), todo esto los hace 
presentar mayores problemas en el secado y en el maquinado, siendo más fáciles o menos difíciles de transformar las especies rojas (De la Paz Pérez, 1985). Dada su estructura, esta madera es difícil de secar, ya que es muy susceptible a varios defectos, si no se hace con prácticas adecuadas de apilado y secado. Estos factores son los que deben estudiarse más para lograr un mejor aprovechamiento de la madera de este género. Aunque existe literatura que trata sobre el tema (Avila 1985, Montes 1985, Fuentes et al. 1996), dada la cantidad de especies y su amplia distribución, aún falta ahondar en el tema.

Por otro lado los encinos rojos presentan un color que va de rosa a rojo y rojizo oscuro, lo que los hacen más atractivos que el color castaño pálido de los blancos (Fig. 7). Sin embargo, en el extranjero, la madera aserrada de los encinos blancos, es muy apreciada.

\section{DISCUSIÓN}

En países europeos como Inglaterra, Francia, Alemania, en donde la utilización de la madera de encinos es amplia, hacen una importante explotación de la madera de encino. A pesar de tener únicamente encinos blancos, hacen selección de las mismas según el uso a las que les destina (Lewington y Streeter, 1993). En Estados Unidos de Norteamérica y Canadá, países con alta explotación de la madera de encino, los criterios de selección son también bastante rigurosos y tienen bien seleccionadas sus especies tanto rojas como blancas e incluso, dentro de estos grupos, toman en cuenta el origen geográfico de la misma, especialmente para fines de exportación (AHEC, 1996).

El aprovechamiento de la madera de encino en México, se hace sin separar las especies rojas de las blancas y por lo tanto sin seleccionar las especies dentro del mismo grupo. Esto ocasiona un gran desperdicio de madera, principalmente de las especies blancas, ya que las técnicas de transformación empleadas no son las adecuadas para ellas, por las diferencias en las características anatómicas y tecnológicas (De la Paz Pérez y Aguilar 1978). En México, que posee el mayor número de especies a nivel mundial, la selección de las especies de encino para su aprovechamiento es un factor obligado, ya que esta falta de selección ha repercutido en el bajo aprovechamiento de esta madera. Otra causa primordial para su escaso aprovechamiento es el poco interés para ensayar nuevas metodologías para transformar este tipo de madera en productos mejores y de mayor calidad. Por lo mismo se ha creado un abandono de dicho recurso, lo que se ha tratado de resolver con la introducción de madera extranjera. Actualmente, México importa gran cantidad de productos de madera de encino de Estados Unidos y Canadá (Morales, 1995), desaprovechando el potencial que los encinos mexicanos pueden ofrecer.

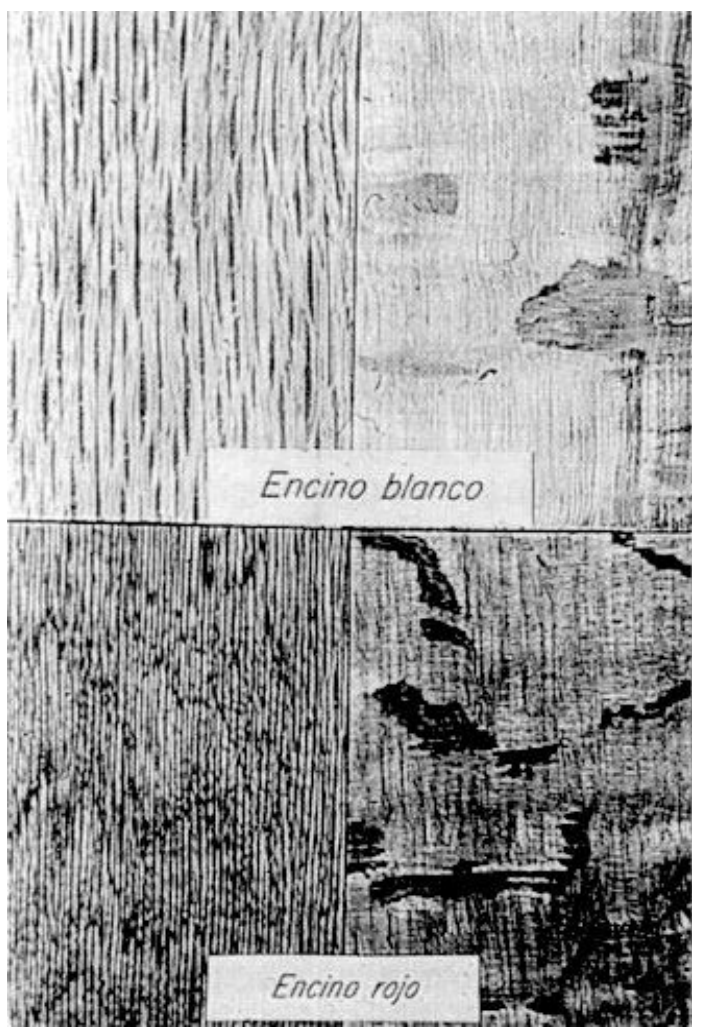

Figura 7. Madera de encino blanco y de encino rojo. 
En los procesos de utilización de otros géneros, se talan indiscriminada e injustificadamente los encinos, ocasionando un alto e inútil desperdicio de este valioso recurso. Si esto, por si sólo es considerable, el deterioro ecológico que se ocasiona a corto y a largo plazo en los bosques, agrava el problema.

La necesidad de conocer a los encinos mexicanos abarca disciplinas como la taxonomía, ecología, en particular sobre sus poblaciones, tecnología, fisiología, mercadotecnia, restauración, etc. El estudio y el conocimiento de las características anatómicas y tecnológicas de la madera, son herramientas valiosas que ayudan a su mejor aprovechamiento y permiten dar a cada especie o grupo de especies, los usos más acordes con sus propiedades (Wangaard, 1981; De la Paz Pérez et al, 1997). Es urgente la necesidad de fomentar programas de conservación, manejo y aprovechamiento de esta importante fuente de materias primas, así como el conocimiento del equilibrio natural de los ecosistemas donde crecen. Es necesario recordar que la explotación silvícola no debe ni puede estar basada en la tala despiadada ni en las vedas totales; la riqueza forestal de un país debe explotarse racional e íntegramente en beneficio del propio sistema ecológico y de la economía nacional. La erosión que afecta el sustrato de los bosques de Quercus tiene efectos a menudo no sólo en la región donde se cortan los árboles, sino también a distancia pues provoca la desecación de manantiales, contaminación de agua, inundaciones, azolve de presas y tolvaneras.

Es tarea urgente buscar la conservación de los encinares, ya que su presencia es necesaria para preservar el equilibrio hidrológico de las cuencas. Para lograrlo, es recomendable ampliar las líneas de investigación orientadas a encontrar más y mejores posibilidades para su utilización y la de otros componentes de los bosques de Quercus, de modo que los propietarios de los terrenos puedan explotar estos bosques con provecho y por ende tengan interés en mantenerlos y conservarlos en buenas condiciones. Por la gran cantidad de especies que existen en México, es urgente una selección y hacer silvicultura.

\section{RECONOCIMIENTOS}

Los autores agradecen al Ing. Jorge Becerra, sus valiosas sugerencias al manuscrito.

\section{REFERENCIAS}

Aguilar E., L., C. de la Paz Pérez O. y E. Guerrero C. 1999. Arboles y arbustos del género Quercus. IV Congreso Mexicano Sobre Recursos Naturales. Universidad Juárez de Dgo, México.

American Hardwood Export Council (AHEC), 1996. Temperate hardwoods of the USA. Specifications. American Hardwood Export Council. Washington, D.C. 30 p.

Avila S., C.G. 1985. Secado en estufa de la madera aserrada de mezcla de encinos blancos y rojos. II Seminario Nacional Sobre Utilización de Encinos. Pub. Esp. Inst. Nac. Invest. For. No. 49:218-228.

Becerra, J. 1999. Encino: características y tendencias de su producción. Informe. Dirección General de Aprovechamiento Forestal. SEMARNAP.

Bravo G., L.R. 1995. El encino como materia prima en la elaboración de carbón vegetal. III Seminario Nacional Sobre Utilización de Encinos, Tomo II: 661-667. Facultad de Ciencias Forestales, Universidad Autónoma de Nuevo León. Linares, N.L. 
De la Paz Pérez O., C. y L. Aguilar E. 1978. Diferencias morfológicas externas y anatómicas de la madera de los encinos blancos y rojos. Bol. Téc. Inst. Nac. Invest. For. No. 59. 19 p

De la Paz Pérez O., C.1985. Variación de la estructura anatómica de los encinos y su efecto en el aprovechamiento de los mismos. II Seminario Nacional Sobre Utilización de Encinos. Pub. Esp. Inst. Nac. Invest. For. No. 49:291312.

De la Paz Pérez O., C., R. Dávalos S. y A. Quintanar I. 1997. Las características tecnológicas de la madera. Contactos 19: 15-21.

De la Paz Pérez O. C. y A. Quintanar I. 1998. Importancia de la madera de encino en la artesanía popular michoacana. ContactoS 25: 29-34.

Fuentes T., F.J, J.A. Silva G. y E. Montes R. 1996. Manual del secado técnico convencional de la madera. Universidad de Guadalajara, Guadalajara, Jal. México. 122 p.

Green, D.W., R.J. Ross y K.A. McDonald. 1993. Production of hardwood machine stress rated lumber. 9th International Symposium on nondestructive testing of wood: 141-150. Forest Products Society, Madison, WI.

Lewington, $R$ y D. Streeter. 1993. La historia natural de los robles. Ediciones B., S.A. Barcelona. 60 p.

McVaugh, R. 1974. Flora novo-galiciana. Contr. Univ. Michigan Herb. 12:1-93.
Montes R., E. 1985. Alternativas para el secado de la madera de encino. II Seminario Nacional Sobre Utilización de Encinos. Pub. Esp. Inst. Nac. Invest. For. No. 49:229-237.

Morales T., C. 1995. Comercio nacional e internacional del encino. Informe. Secretaría de Desarrollo Rural del Estado de Jalisco.

Pennington, T. D. y J. Sarukhán K. 1998. Árboles tropicales de México. Manual para la identificación de las principales especies. UNAM-FCE. México. 521 p.

Rzedowski, J. 1978. Vegetación de México. Limusa. México, D. F. 482 p.

SEMARNAP. 1996. Anuario estadístico de la producción forestal 1996. SEMARNAP - DGF. México, D. F. $125 \mathrm{p}$.

SEMARNAP. 1999. Estadísticas del Sector Forestal. Dirección General Forestal. Año 2, No. 1, Agosto 1999. SEMARNAP - DGF. México, D. F. 4 p.

Trelease, W. 1924. The american oaks. Mem. Nat. Acad. Sci. 20:1-238.

Wangaard, F. F. 1981. Wood: its structure and properties. I. Forest Products Laboratory. EUA. 465 p.

Zavala Ch., F. 1990. Los encinos de México: un recurso desaprovechado. Ciencia y Desarrollo XVI (95):43-51.

Zavala Ch., F. 1995. Encinos y robles, notas fitogeográficas. División de Ciencias Forestales. Universidad Autónoma Chapingo. México. 44 p. 